\title{
1 \\ Optimal control of periodic systems
}

\author{
V. Barbu \\ Department of Mathematics, University of Iaşi \\ 6600 Iaşi, Romania.
}

Phone: (40) 32-213093. Fax: 32-146330. E-mail: barbuQuaic.ro

\begin{abstract}
This work is concerned with existence and maximum principle for optimal control problems with linear periodic state systems in Hilbert spaces.

Keywords

Linear periodic operators, $p$-stabilizable, $p$-detectable, parabolic control systems, periodic wave equation
\end{abstract}

\section{INTRODUCTION}

We shall study here infinite dimensional optimal control problems of the form

Minimize

$\int_{0}^{T}(g(C y(t))+h(u(t))) d t$

subject to $u \in L^{2}(0, T ; U)$ and $y \in C([0, T] ; H)$ solution to state system

$$
\frac{d y}{d t}+A y=B u+f, t \in(0, T) \text {. }
$$

Here $-A$ is the generator of a $C_{0}$-semigroup $e^{-A t}$ on a real Hilbert space $H, B \in L(U, H)$, $C \in L(H, Z)$ and $g: Z \rightarrow \bar{R}=(-\infty,+\infty], h: U \rightarrow \bar{R}$ are lower semicontinous convex functions; $U$ and $Z$ are real Hilbert spaces with the norms $|\cdot|_{U},|\cdot| Z$ and scalar products denoted $(\cdot, \cdot)_{U}$ and $(\cdot, \cdot)_{Z}$, respectively. The norm of $H$ is denoted by $|\cdot|$ and the scalar product by $(\cdot, \cdot)$.

This is the general form of periodic optimal control problems governed by parabolic and hyperbolic linear equations with distributed controllers. Problems of this type arise quite often in applications since the solutions of most dissipative systems with periodic forcing asymptotically converge to a periodic orbit. On the other hand, the performance of a steady state control system can be improved by forcing the control input to vary periodically. As a matter of fact (1) is a singular control system and the most common way to avoid some delicate problems due to resonance phenomenon is to assume that the 
pairs $(A, B)$ and $(A, C)$ are stabilizable and respectively, detectable (see e.g. Da Prato (1987), Bensoussan et al. (1993), Barbu and Pavel (1995)). Though these assumptions are quite natural for infinite horizon control problems they are too restrictive for linear periodic control problems of the form (1). Here we shall weaken them to a closed range property assumption (Definition 1) which seems to be the most convenient framework to treat existence and maximum principle in problem (1) in resonant case. We shall use standard notation for function spaces and assume familiarity with basic results of convex analysis.

\section{LINEAR PERIODIC OPERATORS WITH CLOSED RANGE}

Let $\mathcal{A}: D(\mathcal{A}) \subset L^{2}(0, T ; H) \rightarrow L^{2}(0, T ; H)$ be the linear operator defined by

$$
\begin{aligned}
& \mathcal{A} y=f \text { iff } \int_{0}^{T}\left(\left(y(t), \varphi^{\prime}(t)-A^{*} \varphi(t)\right)+(f(t), \varphi(t))\right) d t=0 \\
& \forall \varphi \in X=\left\{\varphi \in W^{1,2}([0, T] ; H) ; A^{*} \varphi \in L^{2}(0, T ; H), \varphi(0)=\varphi(T)\right\}
\end{aligned}
$$

Here $W^{1,2}([0, T] ; H)$ is the space of absolutely continous functions $\varphi:[0, T] \rightarrow H$ such that $\varphi^{\prime}=\frac{d \varphi}{d t} \in L^{2}(0, T ; H)$. It is readily seen that $\mathcal{A}$ is closed and densely defined. A function $y \in L^{2}(0, T ; H)$ which satisfies (3) is called weak solution to periodic problem

$$
\frac{d y}{d t}+A y=f, t \in(0, T) ; y(0)=y(T) \text {. }
$$

We shall call $\mathcal{A}$ the linear periodic operator associated with $A$. It is readily seen that the adjoint $\mathcal{A}^{*}$ of $\mathcal{A}$ is given by

$$
\mathcal{A}^{*} z=g \text { iff } \int_{0}^{T}\left(\left(z(t), \varphi^{\prime}(t)+A \varphi(t)\right)-(g(t), \varphi(t))\right) d t=0
$$

for all $\varphi \in Y=\left\{\varphi \in W^{1,2}([0, T] ; H) ; A \varphi \in L^{2}(0, T ; H)\right\}$. If the range $R(\mathcal{A})$ of $\mathcal{A}$ is closed in $L^{2}(0, T ; H)$ then we have

$L^{2}(0, T ; H)=R(\mathcal{A}) \oplus N\left(\mathcal{A}^{*}\right), R(\mathcal{A})=N\left(\mathcal{A}^{*}\right)^{\perp}$

and the operator $\mathcal{A}^{-1}$ is continous from $R(\mathcal{A})$ to $R\left(\mathcal{A}^{*}\right)=N(\mathcal{A})^{\perp}$. (Here $N(\mathcal{A})$ and $N\left(\mathcal{A}^{*}\right)$ are the null spaces of $\mathcal{A}$ and $\mathcal{A}^{*}$.) A simple criterion for $R(\mathcal{A})$ to be closed is given in Proposition 1 below which is related to some earlier result of Prüss (1985).

Proposition 1 Assume that for each $m \in Z$, the range $Y_{m}$ of $\mu_{m} i I+A$ is closed in $H$ and

$\sup \left\{\left\|\left(\mu_{m} i I+A\right)^{-1}\right\|_{L\left(Y_{m}, H\right)} ; m \in Z\right\}<\infty$

where $\mu_{m}=2 m \pi T^{-1}$. Then $R(\mathcal{A})$ is closed. 
Proof. We have denoted again $A$ the realization of $A$ in the complexified space $H$. If $f \in R(\mathcal{A})$ and $\mathcal{A} y=f$ then we have

$y(t)=\sum_{m} y_{m} \exp \left(\mu_{m} i t\right), t \in(0, T)$

where $y_{m}=\left(\mu_{m} i+A\right)^{-1} f_{m}$ and $f_{m}=T^{-\frac{1}{2}} \int_{0}^{T} \exp \left(-\mu_{m} i t\right) f(t) d t$. Then by (7) we see that

$\|y\|_{L^{2}(0, T ; H)} \leq C\|f\|_{L^{2}(0, T ; H)}$

which implies that $R(\mathcal{A})$ is closed.

Let $\mathcal{A}_{0}: D\left(\mathcal{A}_{0}\right) \subset L^{2}(0, T ; H) \rightarrow L^{2}(0, T ; H)$ be the linear operator

$A_{0} f=f \quad$ iff $y(t)=e^{-A t} y(T)+\int_{0}^{t} e^{-A(t-s)} f(s) d s, \forall t \in[0, T]$

In other words, $\mathcal{A}_{0} y=f$ if $y$ is a periodic mild solution to equation (4). Clearly $\mathcal{A}_{0}$ is closed and densely defined.

Proposition 2 We have $\mathcal{A}_{0}=\mathcal{A}$.

Proof. It is readily seen that $\mathcal{A}_{0} y=\mathcal{A} y, \forall y \in D\left(\mathcal{A}_{0}\right) \subset D(\mathcal{A})$. We have

$y(t)=\sum_{m \in Z} y_{m} \exp \left(\mu_{m} i t\right)$ in $L^{2}(0, T ; H) ;\left(\mu_{m} i+A\right) y_{m}=f_{m}$.

Then the sequence

$y_{n}(t)=\sum_{|m| \leq n} y_{m} \exp \left(i \mu_{m} t\right)$

is convergent to $y$ in $L^{2}(0, T ; H)$ and for each $n, y_{n}$ is a mild solution to (4) where $f=\sum_{|m| \leq n} f_{m} \exp \left(i \mu_{m} t\right)$. Letting $n \rightarrow+\infty$ we get that $y$ satisfies (10) as claimed.

By Proposition 2 and (9) we have

$$
\begin{aligned}
& R(\mathcal{A})=\left\{f \in L^{2}(0, T ; H) ; \int_{0}^{T} e^{-A(T-t)} f(t) d t \in R\left(I-e^{-A T}\right)\right\} \\
& N(\mathcal{A})=\left\{y \in L^{2}(0, T ; H) ; y(t)=e^{-A t} y_{0},\left(I-e^{-A T}\right) y_{0}=0\right\}
\end{aligned}
$$

and so we have

Corollary 1 If $R\left(I-e^{-A T}\right)$ is closed in $H$ then $R(\mathcal{A})$ is closed in $L^{2}(0, T ; H)$. 
In particular, by Riesz-Fredholm theorem we have

Corollary 2 If $e^{-A T}$ is compact then $R(\mathcal{A})$ is closed and $N(\mathcal{A})$ is finite dimensional.

Note also that by Proposition 2 if $R(\mathcal{A})$ is closed and $y \in L^{2}(0, T ; H)$ is a weak solution to (4) then $y \in C([0, T] ; H)$ and

$\left\|\mathcal{A}^{-1} y\right\|_{C([0, T] ; H)} \leq C\|f\|_{L^{2}(0, T ; H)}, \forall f \in R(\mathcal{A})$.

Note also that if $R\left(I-e^{-A T}\right)$ is closed in $H$ then

$\left\|\mathcal{A}^{-1} y\right\|_{C([0, T] ; H)} \leq C\|f\|_{L^{1}(0, T ; H)}, \forall f \in R(\mathcal{A})$

Moreover, the adjoint operator $\mathcal{A}^{*}$ (see (5),(6)) can be equivalently defined as

$\mathcal{A}^{*} z=g$ iff $z(t)=e^{-A^{*}(T-t)} z(0)+\int_{t}^{T} e^{-A^{*}(s-t)} g(s) d s, \forall t \in[0, T]$.

Throughout in the sequel by solution $y$ to (2) we mean weak solution i.e., a solution to $\mathcal{A} y=f$ (equivalently $\mathcal{A}_{0} y=f$ ).

Definition 1 The pair $(A, B)$ is said to be p-stabilizable if either $R(\mathcal{A})$ is closed and $N(\mathcal{A}), N\left(\mathcal{A}^{*}\right)$ are finite dimensional or there is $F \in L(U, H)$ such that $R\left(I-e^{-(A+B F) T}\right)$ is closed in $H$ and $N\left(\mathcal{A}_{F}\right), N\left(\mathcal{A}_{F}^{*}\right)$ are finite dimensional.

The pair $(A, C)$ is said to be p-detectable if either $R(\mathcal{A})$ is closed and $\operatorname{dim} N(\mathcal{A})<\infty$ or $\exists K \in L(Z, H)$ such that $R\left(I-e^{-(A+K C) T}\right)$ is closed in $H$ and $\operatorname{dim} N\left(\mathcal{A}_{K}\right)<\infty$.

Here $\mathcal{A}_{F}=\mathcal{A}+B F$ and $\mathcal{A}_{K}=\mathcal{A}+K C$. We note that $N(\mathcal{A})$ is finite dimensional if and only if so is $N\left(I-e^{-A T}\right)$.

In particular, $(A, B)$ is $p$-stabilizable in each of the following situations:

1. $(A, B)$ is stabilizable.

2. $R\left(I-e^{-A T}\right)$ is closed and $\operatorname{dim} N\left(I-e^{-A^{*} T}\right)<\infty$. (In particular if $e^{-A T}$ is compact.)

\section{EXISTENCE OF OPTIMAL CONTROLLERS}

We shall study here existence in problem (1) under the following hypotheses:

(i) The pair $(A, C)$ is $p$-detectable and

$$
N(\mathcal{A}) \cap\left\{y \in L^{2}(0, T ; H) ; C y(t)=0 \text { a.e. } t \in(0, T)\right\}=\{0\}
$$

(ii) $g: Z \rightarrow \bar{R}, h: U \rightarrow \bar{R}$ are convex, lower semicontinous and

$$
\begin{aligned}
& g(z) \geq \alpha|z|_{Z}+\beta, \quad \forall z \in Z \\
& h(u) \geq \omega|u|_{U}^{2}+\gamma, \quad \forall u \in U
\end{aligned}
$$


where $\alpha, \omega>0$ and $\beta, \gamma \in R$.

Theorem 1 Under Hypotheses (i), (ii) problem (1) has at least one optimal pair $\left(y^{*}, u^{*}\right) \in C([0, T] ; H) \times L^{2}(0, T ; U)$.

Proof. Let $\left\{\left(y_{n}, u_{n}\right) \in C([0, T] ; H) \times L^{2}(0, T ; U)\right.$ be such that $\mathcal{A} y_{n}=B u_{n}+f$ and

$\inf (1.1)=d<\int_{0}^{T}\left(g\left(y_{n}(t)\right)+h\left(u_{n}(t)\right)\right) d t \leq d+n^{-1}$.

By (17), (18) we have

$\left\|C y_{n}\right\|_{L^{1}(0 . T ; H)}+\left\|u_{n}\right\|_{L^{2}(0, T ; U)} \leq C_{1}$.

By Hypothesis (i) there is $K \in L(Z, H)$ such that $R\left(\mathcal{A}_{K}\right)$ is closed $\left(\mathcal{A}_{K}=\mathcal{A}+K C\right)$, estimate (14) holds and $\operatorname{dim} N(\mathcal{A}+K C)<\infty$. We have

$\mathcal{A}_{K} y_{n}=B u_{n}+K C y_{n}+f$.

We set $y_{n}=y_{n}^{1}+y_{n}^{2}$ where $y_{n}^{1}=\mathcal{A}_{K}^{-1}\left(B u_{n}+K C y_{n}+f\right) \in R\left(\mathcal{A}_{K}^{*}\right)$ and $y_{n}^{2} \in N\left(\mathcal{A}_{K}\right)$. Then by (16) and (20) we have

$\left\|y_{n}^{2}\right\|_{C([0, T] ; H)} \leq C_{2}, \forall n \in N$.

If $R(\mathcal{A})$ is closed we get the same estimate. Then, on a subsequence, again denoted $n$, we have

$$
\begin{aligned}
& u_{n} \quad \longrightarrow \quad u^{*} \quad \text { weakly in } L^{2}(0, T ; U) \\
& y_{n}(t) \longrightarrow y^{*}(t) \text { weakly in } L^{2}(0, T ; H) \text { and } \\
& \text { weakly in } H \text { for } t \in[0, T] \text {. }
\end{aligned}
$$

clearly $\mathcal{A} y^{*}=B u^{*}+f$ and since the convex integrand is weakly lower semicontinous we get

$d=\int_{0}^{T}\left(g\left(y^{*}(t)\right)+h\left(u^{*}(t)\right)\right) d t$

as claimed.

\section{THE MAXIMUM PRINCIPLE}

Here we shall assume that

(j) The pair $(A, B)$ is $p$-stabilizable and

$$
N\left(\mathcal{A}^{*}\right) \cap\left\{p \in L^{2}(0, T ; H) ; B^{*} p(t)=0 \text { a.e. } t \in(0, T)\right\}=\{0\} .
$$


(jj) The function $g: Z \rightarrow R$ is convex and continuous, $h: U \rightarrow \bar{R}$ is convex and lower semicontinuous, int $D(h) \neq \emptyset$.

(jjj) $f(t)=B f_{0}(t)$ where $f_{0} \in C([0, T] ; U)$ and $f_{0}(t) \in \operatorname{int} D(h), \forall t \in[0, T]$.

Here $D(h)=\{u \in U ; h(u)>+\infty\}$.

Theorem 2 Assume that Hypotheses (j), (jj), (jjj) hold. Then the pair $\left(y^{*}, u^{*}\right) \in C([0, T] ; H) \times L^{2}(0, T ; U)$ is optimal in problem (1) if and only if there are $p \in C([0, T] ; H)$ and $\eta \in L^{\infty}(0, T ; Z)$ such that

$$
\begin{aligned}
& \frac{d p}{d t}-A^{*} p=C^{*} \eta, t \in(0, T) ; p(0)=p(T) . \\
& \eta(t) \in \partial g\left(C y^{*}(t)\right), \text { a.e. } t \in(0, T) . \\
& u^{*}(t) \in \partial h^{*}\left(B^{*} p(t)\right), \text { a.e. } t \in(0, T) .
\end{aligned}
$$

Here $\partial g: Z \rightarrow Z$ and $\partial h^{*}: U \rightarrow U$ stand for subdifferentials of $g$ and $h^{*}$ (the conjugate function of $h$ ). The system (23) is considered of course in the weak sense (5) or (15), i.e.,

$\mathcal{A}^{*} p=-C^{*} \eta$

Proof. It is readily seen that equations $(23) \sim(25)$ are sufficient for optimality. To prove necessity we fix an optimal pair $\left(y^{*}, u^{*}\right)$ and consider the approximating control problem

$$
\begin{array}{r}
\operatorname{Min}\left\{\int_{0}^{T}\left(g_{\varepsilon}(C y(t))+\left|y(t)-y^{*}(t)\right|^{2}+\left|u(t)-u^{*}(t)\right|_{U}^{2}+h(u(t))+\varepsilon^{-1}|v(t)|^{2}\right) d t\right. \\
\left.\mathcal{A} y=B u+v+f, u \in L^{2}(0, T ; U), v \in L^{2}(0, T ; H), y \in C([0, T] ; H)\right\}
\end{array}
$$

where

$g_{\varepsilon}(z)=\inf \left\{(2 \varepsilon)^{-1}|z-\bar{z}|_{Z}^{2}+g(\bar{z}) ; \bar{z} \in Z\right\}, \forall z \in Z$

(Recall that $g_{\varepsilon} \in C^{1}(Z)$ is a smooth approximation of $g$.) Since $\mathcal{A}$ is closed it is easily seen that problem (27) has a unique solution $\left(y_{\varepsilon}, u_{\varepsilon}, v_{\varepsilon}\right)$. Moreover, by standard device (see e.g. Barbu (1993)) we get

$$
\begin{array}{llll}
u_{\varepsilon} & \longrightarrow & u^{*} & \text { strongly in } L^{2}(0, T ; U) \\
y_{\varepsilon} \longrightarrow y^{*} & \text { strongly in } L^{2}(0, T ; H) \\
v_{\varepsilon} \longrightarrow & v^{*} & \text { strongly in } L^{2}(0, T ; H)
\end{array}
$$

Next by (27) we have

$$
\int_{0}^{T}\left(\left(C^{*} \nabla g_{\varepsilon}\left(C y_{\varepsilon}\right), z\right)+2\left(y_{\varepsilon}-y^{*}, z\right)+2\left(u_{\varepsilon}-u^{*}, w\right)_{U}+h^{\prime}\left(u_{\varepsilon}, w\right)+2 \varepsilon^{-1}\left(v_{\varepsilon}(t), v(t)\right)\right) d t \geq 0(29)
$$


for all $(z, w, v) \in C([0, T] ; H) \times L^{2}(0, T ; U) \times L^{2}(0, T ; H)$ such that $\mathcal{A} z=B w+v$. We set $p_{\varepsilon}=\varepsilon^{-1} v_{\varepsilon}$. Then $(29)$ yields

$\int_{0}^{T}\left(\left(C^{*} \nabla g_{\varepsilon}\left(C y_{\varepsilon}\right)+2\left(y_{\varepsilon}-y^{*}\right), z\right)+2\left(u_{\varepsilon}-u^{*}, w\right)_{U}+h^{\prime}\left(u_{e}, w\right)+\left(p_{e}, \mathcal{A} z-B w\right)\right) d t \geq 0$,

$\forall z \in D(\mathcal{A}), \forall w \in L^{2}(0, T ; U)$. (Here $h^{\prime}$ is the directional derivative of $h$.) This yields

$\mathcal{A}^{*} p_{\varepsilon}=-C^{*} \nabla g_{\varepsilon}\left(C y_{e}\right)-2\left(y_{\varepsilon}-y^{*}\right)$.

$B^{*} p_{\varepsilon}(t)+2\left(u^{*}(t)-u_{\varepsilon}(t)\right) \in \partial h\left(u_{\varepsilon}(t)\right)$, a.e. $t \in(0, T)$.

We may rewrite the equation

$\mathcal{A} y_{\varepsilon}=B u_{\varepsilon}+f$

as

$\mathcal{A}_{F} y_{\varepsilon}=B u_{\varepsilon}+B F y_{\varepsilon}+f$

where $F \in L(H, U)$ is as in Definition 1 . Then by (28) and Proposition 2 we see that

$y_{e} \longrightarrow y^{*}$ strongly in $C([0, T] ; H)$.

Since $\partial g$ is locally bounded on $H$ and $\nabla g_{\varepsilon} \in \partial g(1+\varepsilon \partial g)^{-1}$ we infer that

$\left|\nabla g_{\varepsilon}\left(C y_{\varepsilon}(t)\right)\right|_{z} \leq C_{3}, \forall t \in[0, T]$.

On the other hand, by assumption (jjj) we have

$h^{*}\left(B^{*} p_{\varepsilon}+2\left(u^{*}-u_{\varepsilon}\right)\right)+\left(f, p_{\varepsilon}\right) \geq$

$\geq \rho\left|B^{*} p_{\varepsilon}\right|_{U}+C_{4}\left|u^{*}-u_{\varepsilon}\right|_{U}-h\left(-f_{0}+\rho\left|B^{*} p_{\varepsilon}\right|_{U}\left|B^{*} p_{\varepsilon}\right|_{U}^{-1}\right)+C_{4}$, a.e. $t \in(0, T)$.

If $\rho$ is sufficiently small, this yields

$$
\begin{aligned}
& \rho \int_{0}^{T}\left|B^{*} p_{\varepsilon}(t)\right|_{U} d t \leq \int_{0}^{T}\left(h^{*}\left(B^{*} p_{\varepsilon}(t)+2\left(u^{*}(t)-u_{\varepsilon}(t)\right)\right)+\left(f(t), p_{\varepsilon}(t)\right)\right) d t+C_{5} \leq \\
& \leq-\int_{0}^{T}\left(\left(\nabla g_{\varepsilon}\left(C y_{\varepsilon}(t)\right), C y_{\varepsilon}(t)\right)_{Z}+h\left(u_{\varepsilon}(t)\right)+2\left(y_{\varepsilon}(t)-y^{*}(t), y_{\varepsilon}(t)\right)\right) d t \leq C_{6}
\end{aligned}
$$

because $\nabla g_{\varepsilon}$ is monotone and $h$ is bounded from below. Here we have also used the equation

$h^{*}(w)+h(u)=(w, u)_{U}, \forall w \in \partial h(u)$.

Hence

$\int_{0}^{T}\left|B^{*} p_{\varepsilon}(t)\right|_{U} d t \leq C_{7}, \forall \varepsilon>0$. 
Now we rewrite $(31)$ as

$\mathcal{A}_{F}^{*} p_{\varepsilon}=-C^{*} \nabla g_{\varepsilon}\left(C y_{\varepsilon}\right)-2\left(y_{\varepsilon}-y^{*}\right)+F^{*} B^{*} p_{\varepsilon}$

and set $p_{\varepsilon}=p_{\varepsilon}^{1}+p_{\varepsilon}^{2}$ where $p_{\varepsilon}^{1} \in R\left(\mathcal{A}_{F}\right)=N\left(\mathcal{A}_{F}^{*}\right)^{\perp}, p_{\varepsilon}^{2} \in N\left(\mathcal{A}_{F}^{*}\right)$. By $(14,(28),(35),(36)$ we have

$\left\|p_{\varepsilon}^{1}\right\|_{C[0, T] ; H)} \leq C_{8}, \forall \varepsilon>0$.

whilst (22) and (36) imply that $\left\{p_{\varepsilon}^{2}\right\}$ is bounded in $L^{2}(0, T ; H)$. Hence selecting further subsequence we have,

$$
\begin{array}{llll}
p_{\varepsilon} & \longrightarrow & \text { weakly star in } L^{2}(0, T ; H) \\
\nabla g_{\varepsilon}\left(C y_{\varepsilon}\right) & \longrightarrow & \eta & \text { weak star in } L^{\infty}(0, T ; Z)
\end{array}
$$

Since $\partial g$ and $\partial h$ are maximal monotone (and therefore weakly-strongly closed) we may pass to limit in (31), (32) to get the optimality system (23)-(25).

The previous result remains true by an easy adaptation of the proof if $B$ is unbounded but is in $L\left(U,\left(D\left(\mathcal{A}^{*}\right)\right)^{\prime}\right)$ and satisfies one of the following two conditions (Lasiecka and Triggiani (1981))

$$
\begin{array}{ll}
\left\|B^{*} e^{-A^{*} t}\right\|_{L(H, U)} \leq C_{9} t^{\gamma-1}, & 0<\gamma<1 \\
\int_{0}^{t}\left|B^{*} e^{-A^{*} s}\right|^{2} d s \leq C_{t}|x|^{2}, & \forall x \in H, t>0 .
\end{array}
$$

In particular, it follows by Theorem 2 that the pair $\left(y^{*}, u^{*}\right)$ is optimal in problem (1) if and only if the dual problem

$\operatorname{Min}\left\{\int_{0}^{T}\left(\tilde{g}(-v(t))+h^{*}\left(B^{*} p(t)\right)+(f(t), p(t))\right) d t ; \mathcal{A}^{*} p=v\right\}$

has a solution $\left(p^{*}, v^{*}\right)$ and $\operatorname{Min}(1)+\operatorname{Min}(39)=0$. (Here $\tilde{g}$ is the concept of $y \rightarrow g(C y)$.) It should be noted that since the pair $\left(A^{*}, I\right)$ is stabilizable the dual problem (39) is simpler than the primal problem (1).

Examples. Throughout in the following $\Omega$ is an open bounded subset of $R^{n}$ with $C^{2}$ boundary $\partial \Omega$.

$1^{\circ}$. Parabolic control problems. Consider the system

$$
\begin{array}{ll}
\frac{\partial y}{\partial t}-\Delta y+b(x) \cdot \nabla y+c(x) y=B u+f(x, t), & (x, t) \in \Omega \times R \\
y(x, t)=0, & \forall(x, t) \in \partial \Omega \times R \\
y(x, t+T)=y(x, t), & \forall(x, t) \in \Omega \times R,
\end{array}
$$

where $b \in W^{1, \infty}\left(\Omega ; R^{n}\right), c \in L^{\infty}(\Omega), f \in L_{\text {loc }}^{2}\left(R ; L^{2}(\Omega)\right)$ is $T$-periodic in $t$ and $B \in$ $L\left(U, L^{2}(\Omega)\right)$ where $U$ is a real Hilbert space (the controller space). 
The operator $A y=-\Delta y+b \cdot \nabla y+c y, D(A)=H_{0}^{1}(\Omega) \cap H^{2}(\Omega)$, generates a $C_{0}$-compact semigroup on $H=L^{2}(\Omega)$.

Then as seen earlier $R(\mathcal{A})$ is closed and $N\left(\mathcal{A}^{*}\right)$ is finite dimensional.

To be more specific we take $U=L^{2}(\Omega)$ and $B u=m u, \forall u \in L^{2}(\Omega)$ where $m \in C(\bar{\Omega})$. Then condition (22) holds. Indeed if $p \in N\left(\mathcal{A}^{*}\right)$ then $p$ is the solution to boundary value problem

$$
\begin{array}{ll}
\frac{\partial p}{\partial t}+\Delta p+\operatorname{div}(b p)-c p=0, & \text { in } \Omega \times R \\
p=0, & \text { in } \partial \Omega \times R
\end{array}
$$

and $B^{*} p=m p$.

Let $\omega_{0}$ be an open subset of $\omega$ such that $m(x) \neq 0, \forall x \in \bar{\omega}_{0}$. We have therefore

$p(x, t)=0, \forall x \in \omega_{0}, t \in R$

and by the unique continuation property of solutions to parabolic equations (see e.g. Saut and Scheurer (1981)) we infer that $p \equiv 0$ as claimed. Hence Theorem 2 is applicable in the present case.

$2^{\circ}$. Linear delay control systems. Consider the delay system

$$
\begin{aligned}
& y^{\prime}(t)+A_{0} y(t)+A_{1} y(t-h)=B_{0} u(t)+f(t) \\
& y(t)=y(t+T) \quad \forall t \in R,
\end{aligned}
$$

where $A_{0}, A_{1}$ are $n \times n$ matrices, $B_{0}$ is a $n \times m$ matrix, $f \in L_{\text {loc }}^{2}\left(R ; R^{n}\right), f(t+T)=f(t)$, $u \in L_{\text {loc }}^{2}\left(R ; R^{n}\right), u(t)=u(t+T)$. It is well known that this system can be written in the form (2) where $H=M_{2}=R^{n} \times L^{2}\left(-h, 0 ; R^{n}\right)$ and

$$
\begin{aligned}
& A\left(y_{0}, y^{0}\right)=\left\{A_{0} y_{0}+A_{1} y^{0}(-h), \frac{d y^{0}}{d s}\right\} \\
& D(A)=\left\{\left(y_{0}, y^{0}\right) \in R^{n} \times W^{1,2}\left([-h, 0] ; R^{n}\right), y_{0}=y^{0}(0)\right\} \\
& B u=\left(B_{0} u, 0\right), u \in R^{m}=U .
\end{aligned}
$$

In virtue of Proposition 1, the corresponding operator $\mathcal{A}$ has closed range in $L^{2}\left(-h, 0 ; R^{n}\right)$. Moreover, $N\left(\mathcal{A}^{*}\right)$ is finite dimensional and if

$$
N\left(m i I-A_{0}^{*}-e^{-m i h} A_{1}^{*}\right) \cap N\left(B_{0}^{*}\right)=\{0\}, \forall m \in Z
$$

then assumption ( $\mathrm{j}$ ) holds and so theorem 2 is applicable. It should be mentioned that condition (40) does not imply the stabilizability of the pair $(A, B)$ as the following example shows

$x_{1}^{\prime}(t)=x_{1}(t)+x_{3}(t-h), x_{2}^{\prime}(t)=x_{2}(t)+x_{3}(t), x_{3}^{\prime}(t)=u(t)$.

This system is not stabilizable (Manitius and Triggiani (1978)). However it is $p$-stabilizable and condition (40) holds. 


\section{THE OPTIMAL CONTROL OF WAVE EQUATION}

The optimal control problem

Minimize

$$
\int_{0}^{T}(g(C y(t))+h(u(t))) d t
$$

subject to $u \in L^{2}(0, T ; H), y \in L^{2}(0, T ; U)$

$$
\begin{aligned}
& y^{\prime \prime}+A y=B u+f, t \in(0, T) \\
& y(0)=y(T), y^{\prime}(0)=y^{\prime}(T)
\end{aligned}
$$

where $A$ is a self-adjoint linear and positively defined operator on the Hilbert space $H$ and $B \in L(U, H)$ can be treated as a special case of problem (1) in the product space $D\left(A^{1 / 2}\right) \times H$. However, a direct approach to this problem allows to weaken hypotheses (i), (j) in this specific case. For simplicity we shall discuss only the maximum principle for problem (41).

By weak solution to equation (42) we mean a function $y \in L^{2}(0, T ; H)$ such that

$\int_{0}^{T}\left(y(t), \varphi^{\prime \prime}(t)+A \varphi(t)\right) d t=\int_{0}^{T}(f(t)+B u(t), \varphi(t)) d t$,

for all $\varphi \in Y=\left\{\varphi \in C^{2}([0, T] ; H) \cap C([0, T] ; D(A)) ; \varphi(0)=\varphi(T), \varphi^{\prime}(0)=\varphi^{\prime}(T)\right\}$. Equivalently,

$\mathcal{W} y=B u+f$

where $\mathcal{W}: D(\mathcal{W}) \subset L^{2}(0, T ; H) \rightarrow L^{2}(0, T ; H)$ is the linear operator defined by

$\mathcal{W} y=f_{0}$ iff $\int_{0}^{T}\left(y(t), \varphi^{\prime \prime}(t)+A \varphi(t)\right) d t=\int_{0}^{T}\left(f_{0}(t), \varphi(t)\right) d t, \forall \varphi \in Y$.

We shall present now two variants of maximum principle for problem (41) involving two different basic assumptions on system (42) and function $h: U \rightarrow \bar{R}$.

Theorem 3 Assume that

(k) $R(\mathcal{W})$ is closed, $\operatorname{dim} N(\mathcal{W})<\infty$ and

$$
N(\mathcal{W}) \cap\left\{p \in L^{2}(0, T ; H) ; B^{*} p(t)=0 \text { a.e. } t \in(0, T)\right\}=\{0\} .
$$

If $h$ and $g$ satisfy Hypotheses (jj), (jjj), and $\partial g$ has sublinear growth, then the pair $\left(y^{*}, u^{*}\right) \in L^{2}(0, T ; H) \times L^{2}(0, T ; U)$ is optimal in problem (41) if and only if there are $p, \eta \in L^{2}(0, T ; H)$ such that

$\mathcal{W} p=-C^{*} \eta, \eta(t) \in \partial g\left(y^{*}(t)\right)$ a.e. $t \in(0, T)$ 
$u^{*}(t) \in \partial h^{*}\left(B^{*} p(t)\right)$ a.e. $t \in(0, T)$

Theorem 4 Assume that

(kk) $R(\mathcal{W})$ is closed and

$\|p\|_{L^{2}(0, T ; H)} \leq M\left\|B^{*} p\right\|_{L^{2}(0, T ; U)}, \forall p \in N(\mathcal{W})$

Then if $g, h$ satisfy assumptions $(\mathrm{jj}), \partial g$ has sublinear growth, $f \in C([0, T] ; H)$ and

$h(u) \leq \alpha_{1}|u|_{U}^{2}+\beta_{1}, \forall u \in U$

the pair $\left(y^{*}, u^{*}\right) \in L^{2}(0, T ; H) \times L^{2}(0, T ; U)$ is optimal in problem (41) if and only if it satisfies system (46),(47).

The proof of Theorem 3 is identical with that of Theorem 2. As regards Theorem 4, if denote by $\left(y_{\varepsilon}, u_{\varepsilon}, p_{\varepsilon}\right)$ the sequence defined in the proof of Theorem 2 where $\mathcal{A}$ is replaced by $\mathcal{W}$ we note that by (49) we have

$\left\|B^{*} p_{\varepsilon}\right\|_{L^{2}(0, T ; U)} \leq C_{10}$

and so by (48) it follows that $\left\{p_{\varepsilon}^{2}\right\}$ is bounded in $L^{2}(0, T ; H)$. From now on the proof is identical with that of Theorem 2 .

$3^{\circ}$. The one dimensional wave equation. Consider the control system

$$
\begin{array}{ll}
y_{t t}(x, t)-v^{-1}(x)\left(v(x) y_{x}(x, t)\right)_{x}=m(x) u(x, t)+f(x, t), & \\
y(0, t)=y(\pi, t)=0, & x \in(0, \pi) \times R \\
y(x, t+T)=y(x, t), y_{t}(x, t+T)=y_{t}(x, t), & (x, t) \in(0, \pi) \times R
\end{array}
$$

where $v \in H^{2}(0, T), v(x)>0, \forall x \in[0, \pi], m \in C([0, T]) m \not \equiv 0$ and

ess $\inf \left\{\left(v^{\prime}(x)\right)^{2}-2 v^{\prime \prime}(x) v(x) ; x \in(0, \pi)\right\}<0$.

In this case $H=L^{2}(0, \pi), A y=-v^{-1}\left(v y_{x}\right)_{x}, D(A)=H_{0}^{1}(0, \pi), U=L^{2}(0, \pi), B u=m u$, $\forall u \in U$. Equation (50) models the forced vibrations of a nonhomogeneous string as well as the propagation of waves in nonisotropic media. If $T$ is a rational multiple of $\pi$ then $R(\mathcal{W})$ is closed and $N(\mathcal{W})$ is finite dimensional (Barbu and Pavel (to appear)). If $y \in N(\mathcal{W})$ and $m(x) y(x, t)=0$ a.e. $(x, t) \in(0, \pi) \times(0, T)$ then $y(x, t)=0$ a.e. $(x, t) \in(\alpha, \beta) \times(0, T)$. Indeed,

$y(x, t)=\sum_{k=1}^{n_{0}} \alpha_{k} e^{i \mu_{k} t} \varphi_{k}(x), \quad \forall(x, t) \in Q=(0, \pi) \times(0, T)$

where $\mu_{k}=2 k \pi / T$ and $\varphi_{k}$ are the eigen functions of the Sturm-Liouville problem associated to operator $y \longrightarrow v^{-1}(x)\left(v(x) y_{x}\right)_{x}$ we infer that $\alpha_{k}=0, \forall k$. Hence assumption (k) holds in this case. 
$4^{\circ}$. The $n$-dimensional wave equation. Consider the control system

$$
\begin{aligned}
& y_{t t}-\Delta y=m(x) u+f, x \in \Omega, t \in R \\
& y=0, \text { in } \partial \Omega \in R ; y(x, t+T)=y(x, t), y_{t}(x, t+T)=y_{t}(x, t)
\end{aligned}
$$

where $\Omega=(0, \pi)^{n}, m \in C(\bar{\Omega}), m \not \equiv 0$ and $f \in C\left([0, T] ; L^{2}(\Omega)\right), f(x, t+T) \equiv f(x, t)$. We may write (52) in the form (42) where $H=L^{2}(\Omega), A=-\Delta, D(A)=H_{0}^{1}(\Omega) \cap H^{2}(\Omega)$ and $B u=m u, \forall u \in U=L^{2}(\Omega)$.

If $T$ is a rational multiple of $\pi$ then the corresponding operator $\mathcal{W}: L^{2}(Q) \rightarrow L^{2}(Q)$, $Q=\Omega \times(0, T)$ has closed range. Here is the argument (see also N.Pavel (to appear)). If

$\mathcal{W} y=f,(y, f) \in L^{2}(Q) \times L^{2}(Q)$

then

$y=\sum_{m \in Z, k \in N^{n}} f_{m k}\left(\mu_{m}^{2}-\lambda_{k}^{2}\right)^{-1} e^{i \mu_{m} t} \varphi_{k}$

where $\mu_{m}=2 m \pi T^{-1}, \lambda_{k}^{2}=k_{1}^{2}+k_{2}^{2}+\cdots+k_{n}^{2}, k_{i} \in N$ are the eigenvalues of $A$ and $\varphi_{k}$ are the corresponding eigen functions; $f_{m k}$ are the Fourier coefficients of $f$. If $T$ is a rational multiple of $\pi$ we see by (53) that

$\|y\|_{L^{2}(Q)} \leq C_{11}\|f\|_{L^{2}(Q)}, \forall f \in R(\mathcal{W})$

as claimed.

Let us check now condition (48). If $\left\{p_{\varepsilon}\right\} \subset L^{2}\left(0, T ; L^{2}(\Omega)\right)$ is in $N(\mathcal{W})$, i.e., $\mathcal{W} p_{\varepsilon}=0$ and $\left\{m p_{\varepsilon}\right\}$ is bounded in $L^{2}\left(0, T ; L^{2}(\Omega)\right)$ we have for some interval $(a, b) \subset(0, \pi)$

$$
\begin{array}{ll}
\int_{a}^{b} d x \int_{0}^{T} p_{\varepsilon}^{2}(x, t) d x d t \leq C_{12}, & \forall \varepsilon>0 \\
p_{\varepsilon}(x, t)=\sum_{k} a_{\varepsilon k} e^{i \mu_{k} t} \varphi_{k}(x), & \forall(x, t) \in Q
\end{array}
$$

where $\left|\mu_{k}\right|=\lambda_{k}$. By (54) we get

$\sum_{k} a_{\varepsilon k}^{2} \int_{a}^{b} \varphi_{k}^{2}(x) d x \leq C_{13}, \forall \varepsilon>0$.

Hence

$\sum_{\left|\mu_{k}\right|=\lambda_{k}} a_{\varepsilon k}^{2}<C_{14}, \forall \varepsilon>0$

and this implies that $\left\{p_{\varepsilon}\right\}$ is bounded in $L^{2}((0, T) \times \Omega)$ as claimed.

Remark. In particular, the cost functional $(1)$ in $L^{2}(Q)$ is of the form

$\int_{Q} g_{0}(x, y(x, t)) d x d t+\int_{0}^{T} h(u(t)) d t$ 
where $g_{0}: \Omega \times R \rightarrow R$ is continuous, convex and has quadratic growth in $y$. In the special case $m=1$ the maximum principle for this problem follows for a general domain $\Omega$ under assumption (jjj) as a consequence of Theorem 2 because the pair $(A, B)$ is stabilizable. In the case $n=1$ assumption (jjj) can be weakened to an interiority condition in $L^{\infty}(\Omega)$ (Barbu (to appear)). The general case $\Omega \subset R^{n}$ and $m \equiv 1$ is open.

\section{REFERENCES}

Barbu, V. (1993) Analysis and control of nonlinear infinite dimensional systems. Academic Press, Boston.

Barbu, V. (to appear) Optimal control of the one dimensional periodic wave equation. Applied Mathematics and Optimization.

Barbu, V. and Pavel, N. (1995) Optimal control of periodic systems. Applied Mathematics and Optimization.

Barbu, V and Pavel, N. (submitted) Periodic solutions to nonlinear one dimensional wave equation with $x$-dependent coefficients.

Bensoussan, A., Da Prato, G, Delfour, M., Mitter, S.K., (1993) Representation and control of infinite dimensional control systems. Birkhäuser, Boston, Basel, Berlin.

Da Prato, G., (1987) Synthesis of optimal control for an infinte dimensional periodic problem. SIAM Journal on Control and Optimization, 25, 706-14.

Lasiecka, I. and Triggiani, R. (1991) Algebraic Riccati equations with applications to boundary joint control problems. Continuous theory and approximation theory. LNCIS. Springer Verlag, Berlin, New York.

Prüss, J. (1984) On the spectrum of $C_{0}$-semigroup. Transactions American Mathematical Society, 284, 847-57.

Manitius, A. and Triggiani, R. (1978) Function space controllability of linear retarded systems: a derivation from abstract operator condition. SIAM Journal on Control 16, 599-645.

Pavel, N., (to appear) Periodic solutions to nonlinear $2-D$ wave equations.

Saut, C. and Scheurer, B. (1987) Unique continuation for some evolution equations. Journal Differential Equations, 16, 118-39. 